\title{
Does parton saturation at high density explain hadron multiplicities at RHIC ?
}

\author{
R. Baier ${ }^{a}$, A.H. Mueller ${ }^{b}$, D. Schiff ${ }^{c}$, and D.T. Son ${ }^{d}$ \\ ${ }^{a}$ Fakultät für Physik, Universität Bielefeld, D-33501 Bielefeld, Germany \\ ${ }^{b}$ Department of Physics, Columbia University, New York, NY 10027, USA \\ ${ }^{c}$ LPT, Université Paris-Sud, Bâtiment 210, F-91405 Orsay, France \\ ${ }^{d}$ Institute for Nuclear Theory, University of Washington, Seattle, WA 98195-1550, USA
}

\begin{abstract}
We discuss the recent claim that hadron multiplicities measured at RHIC energies are directly described in terms of gluon degrees of freedom fixed from the initial conditions of central heavy ion collisions. The argument is based on the parton saturation scenario expected to be valid at high parton densities and on the assumption of conserved gluon number. Alternatively we conjecture that "bottom-up" equilibration before hadronization modifies this picture, due to nonconservation of the number of gluons.
\end{abstract}

At RHIC and LHC energies in the central rapidity region of heavy ion collisions a high density of energy is deposited mainly in the form of gluons. In recent papers by Kharzeev et al. [1] (hereafter referred to as KLN) it is assumed that the initial gluon density determines the number of produced (charged) particles, i.e. that there is a direct correspondence between the number of partons in the initial state and the number of particles in the final state. The argument is formulated in the framework of the saturation scenario [5] 9], which determines the initial gluon distribution inside the colliding nuclei, and where the characteristic momentum scale is the hard scale $Q_{s}>>\Lambda_{Q C D}$.

Based on this scenario the initial gluon multiplicity immediately after the high energy nuclear collisions can be calculated in the McLerran-Venugopalan model [8 11]. At $\tau_{0} \simeq 1 / Q_{s}$ this initial gluon density is [11],

$$
n_{\text {hard }}\left(\tau_{0}\right)=c \frac{\left(N_{c}^{2}-1\right) Q_{s}^{3}}{4 \pi^{2} N_{c} \alpha_{s}\left(Q_{s} \tau_{0}\right)} .
$$

In 113 this relation is used to obtain the hadron multiplicities in A-A collisions, which are then compared with the corresponding RHIC data [12 16].

In this note we propose a possibly more realistic description, which departs from taking the initial condition as the only one determining ingredient for the final hadronic state. This description is based on the "bottom-up" scenario [17 19], which leads to thermalization of the gluons produced after the collision. In contrast to KLN, this scenario stresses the importance of branching processes of gluons which may allow for a short enough equilibration time, at least at high energies. As a consequence the number of gluons is increasing between initial and equilibration times. In this context let us note that already in [20] a phenomenological factor $\kappa_{\text {inel }}>1$ is introduced accounting for gluon number changing processes which may occur at late times beyond when the classical approach is applicable.

In the following we investigate in the "bottom-up" picture the resulting hadron multiplicities and compare with the predictions of the KLN model. We discuss in some detail the conceptual differences between these two interesting possibilities.

The arguments of KLN [1] go through a number of steps, which we first critically review:

i) The number of gluons in the initial state, and at the time when gluons transform into hadrons, is assumed to be equal, which can be true when only $2 \leftrightarrow 2$ processes are taken into account. Only in this circumstance the measured hadron multiplicities are reflecting directly the initial conditions.

ii) In (11) the parameter $c$ is a constant linking the number of gluons in the nucleus wave function to the number of gluons which are freed during the collision. It is expected to be of $O(1)$ [11]. An (approximate) analytical calculation gives $c=2 \ln 2 \simeq 1.39$ [21. However, extracting $c$ from the numerical simulation in [9,20] leads to the estimate 1$]$ $c \simeq 0.5$ for $Q_{s}=1 \mathrm{GeV}$.

iii) The hard saturation scale $Q_{s}^{2}$ in the case of one nucleus has been determined to be [11],

$$
Q_{s}^{2}(\vec{s})=\frac{4 \pi^{2} N_{c}}{N_{c}^{2}-1} \alpha_{s}\left(Q_{s}^{2}\right) x G\left(x, Q_{s}^{2}\right) \rho_{\text {nucleon }}(\vec{s}),
$$

${ }^{1}$ This estimate is based on the relation $c=4 \pi^{2} f_{N} /\left[\left(N_{c}^{2}-1\right) \ln Q_{s}^{2} / \Lambda_{Q C D}^{2}\right]$, where $f_{N}=0.322$. 
where $\vec{s}$ is the impact parameter, $x G\left(x, Q^{2}\right)$ is the gluon structure function in the nucleon, and $\rho_{\text {nucleon }}$ is the transverse density of nucleons in the nucleus. Notice that in the above equation an additional numerical multiplicative factor may come as a consequence of the inherent uncertainty in the precise determination of the saturation momentum outside of the McLerran-Venugopalan model [8]. We shall call this factor $K$ and introduce it a little later on. KLN generalize (2) to the case of two colliding nuclei. Let us first write 2

$$
Q_{s}^{2}(\vec{s}, \vec{b})=\frac{4 \pi^{2} N_{c}}{N_{c}^{2}-1} \alpha_{s}\left(Q_{s}^{2}\right) x G\left(x, Q_{s}^{2}\right) \frac{\rho_{\text {part }}(\vec{s}, \vec{b})}{2} .
$$

where $\rho_{\text {part }}(\vec{s}, \vec{b})$ is the density of participating nucleons in the transverse plane as a function of the impact parameter $\vec{b}$ of the A-A collision, and of $\vec{s}$ the transverse coordinate of the produced gluon. The factor $\frac{1}{2}$ is required by the proper definition of $Q_{s}^{2}$, relative to one nucleus [1]. Integrating the density with respect to $\vec{s}$ leads to

$$
\int d^{2} s \rho_{\text {part }}(\vec{s}, \vec{b})=N_{\text {part }}(\vec{b})
$$

with $N_{\text {part }}(\vec{b})$ the number of participants in the A-A collision at fixed $\vec{b}$.

iv) Focusing on the distribution of freed gluons $d N / d \eta$ for $\eta=0$ at given $\vec{b}$, we can write

$$
\begin{aligned}
\frac{d N}{d \eta}(\vec{b}) & =c \frac{N_{c}^{2}-1}{4 \pi^{2} N_{c}} \int d^{2} s \frac{1}{\alpha_{s}} Q_{s}^{2}(\vec{s}, \vec{b}) \\
& \simeq c x G\left(x, \bar{Q}_{s}^{2}\right) \frac{N_{\text {part }}(\vec{b})}{2} .
\end{aligned}
$$

The corresponding charged hadron multiplicity for the most central collisions is then obtained as

$$
\left\langle\frac{2}{N_{\text {part }}} \frac{d N_{c h}}{d \eta}\right\rangle \simeq \frac{1}{3} c\left[\ln \frac{\bar{Q}_{s}^{2}}{\Lambda_{Q C D}^{2}}\right],
$$

using the gluon structure function (8) given below. As reference energy we take $\sqrt{s}=130 \mathrm{GeV}$.

One should remark that in (5) and (6), $\bar{Q}_{s}^{2}$ shows up as an effective average over the variable $\vec{s}$ in (3). As a first approximation the following is used:

$$
\bar{Q}_{s}^{2}(\vec{b})=K \frac{4 \pi^{2} N_{c}}{N_{c}^{2}-1} \alpha_{s}\left(\bar{Q}_{s}^{2}\right) x G\left(x, \bar{Q}_{s}^{2}\right) \frac{\rho_{\text {part }}(\vec{b})}{2} .
$$

v) Information on the gluon structure function is necessary. In the small $x$ regime, the main feature is that it increases with $Q^{2}$ at fixed $x$. Following KLN [1] [3], it is reasonable to take

$$
x G\left(x, Q^{2}\right)=0.5 \ln \left(\frac{Q^{2}}{\Lambda_{Q C D}^{2}}\right),
$$

with $\Lambda_{Q C D}=200 \mathrm{MeV}$, such that $x G\left(x, Q^{2}\right) \simeq 2$ at $Q^{2}=2 \mathrm{GeV}^{2}($ at $x=0.02)$.

vi) The strong coupling constant is

$$
\alpha_{s}\left(Q^{2}\right) \simeq \frac{1}{\beta_{0} \ln \left(\frac{Q^{2}}{\Lambda_{Q C D}^{2}}\right)},
$$

with $\beta_{0}=\left(11-2 n_{f} / 3\right) / 4 \pi$ for $N_{c}=3$; we take $n_{f}=3$.

vii) Using (7) together with the above choices for $\alpha_{s}$ and $x G\left(x, Q^{2}\right)$, one finds for central $A u-A u$ collisions at RHIC (at $\sqrt{s}=130 \mathrm{GeV}$ ), taking for the moment $K=1$ :

$$
\bar{Q}_{s}^{2}(\vec{b}=0) \simeq 0.63 \mathrm{GeV}^{2} \text {, using } \rho_{\text {part }}(\vec{b}=0)=3.06 \mathrm{fm}^{-2},
$$

\footnotetext{
${ }^{2}$ Notice in (3), consistently with (2), the factor $\frac{1}{2}$ compared to eq. (14) in [1]. This has the consequence of lower values of $Q_{s}^{2}$ appearing in our discussion of the KLN approach.
} 
as quoted in Table 2 by [1]. For larger values of $\vec{b}, \bar{Q}_{s}^{2}$ becomes even smaller, e.g. for $b=10 \mathrm{fm}, \bar{Q}_{s}^{2} \simeq 0.32 \mathrm{GeV}^{2}$ ! This casts some doubts on the applicability of this model, even for central collisions at RHIC energies.

As already noticed, the missing factor $\frac{1}{2}$ in KLN and a larger value used for $\alpha_{s}$, namely $\alpha_{s}\left(Q^{2}\right)=0.6$ at $Q^{2}=2 \mathrm{GeV}^{2}$, allows them to get larger values of $Q_{s}^{2}$, i.e. $Q_{s}^{2} \geq 2 \mathrm{GeV}^{2}$ for the central collisions.

Taking in the following an optimistic point of view, we use (7) with a multiplicative $K$ factor of order $O(1)$, explicitely $K \simeq 1.6$, such that for $\vec{b}=0, \bar{Q}_{s}^{2}(\vec{b}=0)=1 \mathrm{GeV}^{2}$. Our use of $K$ is to a large extent cosmetic. This factor only appears in calculating $Q_{s}^{2}$, but does not change (6) or (18). Such a factor will affect the average transverse momentum per produced gluon but not the total number of produced gluons. The difference between $\bar{Q}_{s}(\vec{b}=0)=1 \mathrm{GeV}$ and $\bar{Q}_{s}(\vec{b}=0)=0.8 \mathrm{GeV}$, corresponding to $K=1.6$ and $K=1$, respectively, has little effect on the equilibration temperature $T_{e q}$ and time $\tau_{e q}$ quoted later in this note.

viii) Finally, there is an equality assumed between the number of partons in the final state and the number of observed hadrons ("parton-hadron duality" 23]).

Let us now turn to the "bottom-up" scenario [17 19], which in contrast to the KLN [1] 3] prescription, does not relate the multiplicities to the initial condition only, but also to the way gluons are thermalized.

In the framework of perturbative QCD the time evolution of the gluonic system, when described by a non-linear Boltzmann equation based on $2 \leftrightarrow 2$ processes, a relatively long time $\left(\sim Q_{s}^{-1} \exp \left(\alpha_{s}^{-1 / 2}\right)\right)$ is required for the approach to kinetic equilibration [11,24]. Fast and efficient thermalization occurs when inelastic processes, namely gluon splittings $2 \leftrightarrow 3$ are taken into account, and kinetic equilibration occurs much faster at times $\sim \alpha_{s}^{-13 / 5} Q_{s}^{-1}$ 17 [19. In this "bottom-up" scenario the time evolution of the system proceeds through several regimes (Fig. 1), with $Q_{s} \tau \sim \alpha_{s}^{-3 / 2}, \alpha_{s}^{-5 / 2}$ and $\alpha_{s}^{-13 / 5}$.

The difference between the "bottom-up" and the KLN picture is schematically illustrated in this Fig. 1. Under the KLN assumption the hard gluons (on the momentum scale $Q_{s}$ ) are conserved in number, i.e. $n_{\text {hard }} \tau=$ const, and they finally hadronize, after passing through a hydrodynamical stage. The "bottom-up" scenario is characterized by the fact that hard gluons are degrading, soft ones are formed and start to dominate the system. As a result the interactions of gluons in this kinetic scenario modify strongly the initial gluon spectrum. The gluons are redistributed and thermalizing, such that a quark gluon plasma is formed. The number of gluons, together with the entropy [19], is increasing with proper time $\tau$, such that the ratio is,

$$
R=\left.\left[n_{\text {soft }}(\tau)\left(Q_{s} \tau\right)\right]\right|_{\tau_{\text {eq }}} /\left.\left[n_{\text {hard }}(\tau)\left(Q_{s} \tau\right)\right]\right|_{\tau_{0}} \sim \alpha_{s}^{-2 / 5} \gg 1 .
$$

The following processes are expected to be present (at RHIC and higher energies in the central region of pseudorapidity $\eta \leq 1)$ : at $Q_{s} \tau \geq 1$ saturated hard gluons $\longrightarrow$ elastic scatterings and branching/production of soft gluons $\longrightarrow$ at $\left.Q_{s} \tau\right|_{e q} \sim \alpha_{s}^{-13 / 5}$ thermalization of these soft gluons with temperature $T_{e q} \sim \alpha_{s}^{2 / 5} Q_{s} \longrightarrow$ hydrodynamic expansion $\longrightarrow$ hadronization (Fig. 国).

In more detail the parametrically estimated time scales are determined as follows [17 [19].

Early times $1 \ll Q_{s} \tau \ll \alpha_{s}^{-3 / 2}$ :

At the earliest time, $\tau \sim Q_{s}^{-1}$, gluons, i.e. hard gluons, have typical large transverse momentum of order $Q_{s}$ and occupation number of order $1 / \alpha_{s}$. Later on gluons with smaller momenta, but still larger than $\Lambda_{Q C D}$ will be produced (nevertheless, they are denoted as soft gluons). The density of hard gluons $n_{\text {hard }}$ decreases with time due to the one-dimensional expansion. Gluons interact by elastic scatterings at small angle, with exchange momentum $\ll Q_{s}$. The typical occupation number is large until $Q_{s} \tau \sim \alpha_{s}^{-3 / 2}$, when it becomes of $O(1)$.

This regime is the transition region from the non-linear classical gluon field to the one where the transport description by Boltzmann equations should become applicable.

Times $\alpha_{s}^{-3 / 2} \ll Q_{s} \tau \ll \alpha_{s}^{-5 / 2}$ :

Inelastic scatterings produce (soft) gluons $n_{\text {soft }}$ with characteristic momentum estimated to be of order $\alpha_{s}^{1 / 2} Q_{s}>$ $\Lambda_{Q C D}$ (Fig. 1), namely via hard + hard $\rightarrow$ hard + hard + soft. The number of these soft gluons becomes comparable to that of hard ones at $Q_{s} \tau \sim \alpha_{s}^{-5 / 2}$, namely $n_{\text {soft }} \sim n_{\text {hard }}$. 


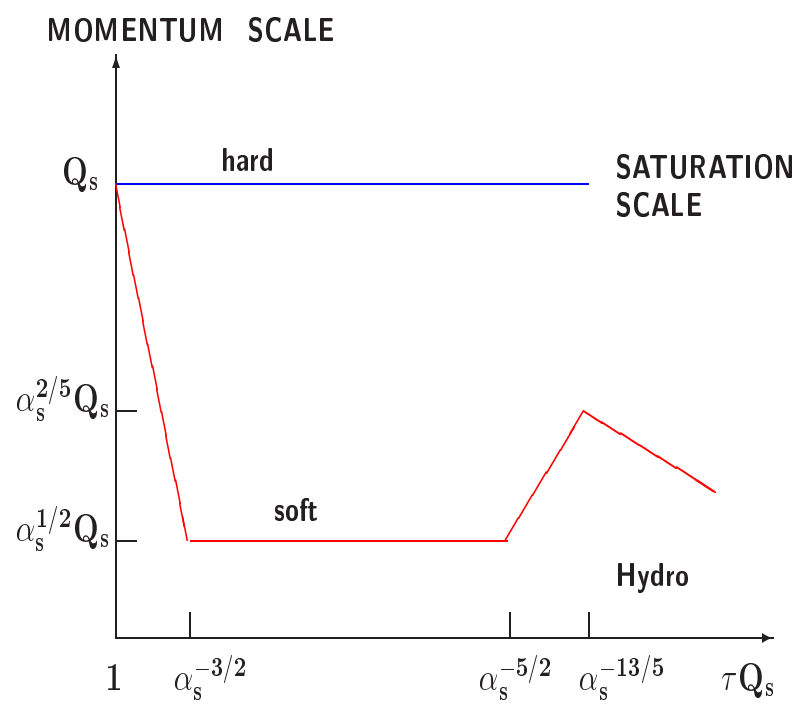

FIG. 1. Characteristic momentum scales for the "bottom-up" scenario.

Times $Q_{s} \tau \gg \alpha_{s}^{-5 / 2}$ :

After $Q_{s} \tau \sim \alpha_{s}^{-5 / 2}$ most gluons are soft, $n_{s o f t} \gg n_{\text {hard }}$; they achieve thermal equilibration amongst themselves. Although the whole system is still not in thermal equilibrium, the soft part is characterized by the temperature $T$, $n_{\text {soft }} \sim T^{3}$. The few hard gluons collide with the soft ones of the thermal bath and constantly loose energy to the latter. A hard gluon emits one with a softer energy, which splits into gluons with comparable momenta. The products of this branching quickly cascade further, giving all their energy to the thermal bath. This increases the temperature in the thermal bath found to be,

$$
T=c_{T} \alpha_{s}^{3} Q_{s}^{2} \tau,
$$

i.e. it increases linearly with time, even when the system is expanding, due to the hard gluons which serve as an energy source. $c_{T}$ is a numerical constant to be discussed later. One can verify a posteriori that soft gluons are indeed equilibrated due to many interactions: the size of the expanding system of $O(\tau)$ is indeed much larger than the mean free path of the soft gluons, i.e.

$$
\tau / \lambda_{\text {soft }} \sim \tau n_{\text {soft }}(\tau) \sigma \sim c_{T} \alpha_{s}^{5}\left(Q_{s} \tau\right)^{2} \gg 1
$$

where the cross section is estimated as $\sigma \sim \alpha_{s}^{2} / T^{2}$.

In the following, in order to provide predictions for particle multiplicities in this scenario we go as much as possible beyond the parametric estimates, ending up with consistency arguments which specify the allowed range of parameters.

First we start by considering the constant $c_{T}$ which may be written in terms of the parameter $c$ as [17],

$$
c_{T} \simeq \frac{15}{8 \pi^{5}} c N_{c}^{3} \simeq 0.16 c .
$$

The linear growth of $T$ as shown in Fig. 1 terminates, when the hard gluons loose all of their energy, i.e. when

$$
\tau=\tau_{e q}=c_{e q} \alpha_{s}^{-13 / 5} Q_{s}^{-1},
$$

where the parameter $c_{e q}$ is unknown for the moment, although in principle it could be calculated in the "bottom-up" framework. The temperature achieves a maximal value, i.e. the equilibration temperature, which is expressed by 


$$
T_{\max }=T_{e q}=0.16 c c_{e q} \alpha_{s}^{2 / 5}\left(Q_{s}^{2}\right) Q_{s} .
$$

Subsequently the temperature decreases as $\tau^{-1 / 3}[25]$, such that $\tau n_{\text {soft }}(\tau)=$ const.

We now derive the charged hadron multiplicity from the number density of the equilibrated soft gluons,

$$
n_{\text {soft }}\left(\tau_{e q}\right)=2\left(N_{c}^{2}-1\right) \frac{\zeta(3)}{\pi^{2}} T_{e q}^{3},
$$

which is much larger than $n_{\text {hard }}$ in (11) used by KLN. One finds for the ratio $R$ defined in (10),

$$
\begin{aligned}
R & =\left.8 \zeta(3) N_{c} \frac{c_{T}^{3}}{c} \alpha_{s}^{10}\left(Q_{s}^{2}\right)\left(Q_{s} \tau\right)^{4}\right|_{\tau_{e q}} \\
& \simeq 0.13 c^{2} c_{e q}^{4} \alpha_{s}^{-2 / 5}\left(Q_{s}^{2}\right) .
\end{aligned}
$$

For the charged hadron multiplicity the result for $\sqrt{s}=130 \mathrm{GeV}$ and the most central collisions, is

$$
\begin{aligned}
\left\langle\frac{2}{N_{\text {part }}} \frac{d N_{c h}}{d \eta}\right\rangle & \simeq \frac{R c}{3} \ln \frac{\bar{Q}_{s}^{2}}{\Lambda_{Q C D}^{2}} \\
& \simeq 0.04 c^{3} c_{e q}^{4}\left[\ln \frac{\bar{Q}_{s}^{2}}{\Lambda_{Q C D}^{2}}\right]^{7 / 5},
\end{aligned}
$$

which replaces (6).

In order to compare the predicted charged hadron multiplicity, (6) and (18), with RHIC data we use as a reference the result by the PHOBOS Collaboration [13], namely $3.24 \pm 0.1$ (stat) \pm 0.25 (syst), which is in good agreement within errors with the experimental measurements of the other collaborations at RHIC. This is best seen by comparing the values for $d N_{c h} / d \eta$ at midrapidity at $\sqrt{s}=130 \mathrm{GeV}: d N_{c h} / d \eta=555 \pm 12($ stat) \pm 35 (syst) [13], $609 \pm 1$ (stat) \pm 37 (syst) [14], $549 \pm 1$ (stat) \pm 35 (syst) [15], $567 \pm 1$ (stat) \pm 38 (syst) [16], respectively.

As reference value we take

$$
\left\langle\frac{2}{N_{\text {part }}} \frac{d N_{c h}}{d \eta}\right\rangle=3.24
$$

First we note that in the KLN approach agreement with experimental data can only be achieved with the value $c \simeq 3$, which is different from the current numerical estimate quoted before, $c \simeq 0.5$.

Turning to the "bottom-up" approach, the experimental value (19) meets the theoretical expectation (18) for $R c \simeq 3$, or equivalently

$$
c_{e q} \simeq \frac{2.0}{c^{3 / 4}} .
$$

This relation is to be confronted with the consistency requirement for the "bottom-up" scenario, that the ratio $R$ (17) be larger than 2, implying

$$
c^{2} c_{e q}^{4} \geq 10, \text { i.e. } c_{e q} \geq \frac{1.8}{\sqrt{c}} .
$$

It is not too difficult to see that (20, 21) constrain the two parameters:

$$
c \leq 1.5 \text { and } c_{e q} \geq 1.5 .
$$

This in turn allows us to infer the actual properties of the medium, especially to answer tentatively the question of the formation of the equilibrated plasma. We do this via discussing the temperature $T_{e q}$ and the equilibration time $\tau_{e q}$. In order that the quark gluon plasma is produced, $T_{e q}$ should be bigger than the phase transition temperature $T_{\text {deconf }}$, which is of order $T_{\text {deconf }}=173 \pm 8 \mathrm{MeV}$ and $154 \pm 8 \mathrm{MeV}$ for 2 and 3 flavour QCD, respectively [26], i.e.

$$
T_{e q} \geq T_{\text {deconf }} .
$$


This constrains

$$
c c_{e q}>1.3 \text {, or } c>0.2 \text {. }
$$

Finally, we may correlatively discuss $\tau_{e q}$. Under the condition (22) one finds (for central collisions)

$$
\tau_{e q} \geq 2.6 \mathrm{fm} \sim 1 / 2 R_{A u},
$$

which is much bigger than the current estimate of $\simeq 0.7 \mathrm{fm} 10$ [ ${ }^{3}$. Within the uncertainties inherent to these estimates the formation of the equilibrated plasma may indeed be realized.

One may consider the energy dependence at RHIC energies of the charged multiplicities. Following [3] it is controlled by the energy dependence of the saturation scale, i.e. $Q_{s}^{2}(s) / Q_{s}^{2}\left(s_{0}\right)=\left(s / s_{0}\right)^{\lambda / 2}$ with $\lambda=0.25$ [28. As discussed we choose as the reference value $\bar{Q}_{s}^{2}\left(s_{0}\right)=1 \mathrm{GeV}^{2}$ at $\sqrt{s_{0}}=130 \mathrm{GeV}$. For the most central collisions the expression for the energy dependence of the pseudorapidity density of charged particles at midrapidity reads in the "bottom-up" scenario,

$$
\left\langle\frac{2}{N_{\text {part }}} \frac{d N_{c h}}{d \eta}\right\rangle \simeq 0.64\left(\frac{\sqrt{s}}{\sqrt{s}_{0}}\right)^{\lambda}\left[\ln \frac{\bar{Q}_{s}^{2}(s)}{\Lambda_{Q C D}^{2}}\right]^{7 / 5}
$$

Based on (26) the multiplicity at $\sqrt{s}=200 \mathrm{GeV}$ is obtained:

$$
\left\langle\frac{2}{N_{\text {part }}} \frac{d N_{c h}}{d \eta}\right\rangle=3.84
$$

compared to the value given by the PHOBOS Collaboration [13],

$$
\left\langle\frac{2}{N_{\text {part }}} \frac{d N_{c h}}{d \eta}\right\rangle=3.78 \pm 0.25 \text { (syst) . }
$$

Finally we consider the centrality dependence, i.e. the dependence of $d N_{c h} / d \eta$ as a function of $N_{\text {part }}$. In Fig. 2 the comparison of the "bottom-up" expectation as derived from (26) with data from the PHOBOS Collaboration 13 at $\sqrt{s}=130 \mathrm{GeV}$ and at $\sqrt{s}=200 \mathrm{GeV}$ at RHIC is shown. For this comparison $\bar{Q}_{s}^{2}(\vec{b})$ is calculated from $(\nabla)$ as a function of $N_{\text {part }}$, using $\rho_{\text {part }}(\vec{b})$ given in Table 2 of [1], together with the scaling relation for the energy dependence of $Q_{s}$ quoted above 28]. One has to note, that for $N_{\text {part }}<100$ the values of $Q_{s}^{2}$ are becoming smaller than $0.6 G^{2} V^{2}$. The structure of the shape of $d N_{c h} / d \eta$ as a function of $N_{\text {part }}$ seen in the data (Fig. 2) could be attributed, cf. with (5), to details of $x G\left(x, Q_{s}^{2}\right)$ at small $x$ as a function of $Q_{s}^{2}$.

\footnotetext{
${ }^{3}$ This short equilibration time is required in order to describe the hadron spectra for different particles as measured at RHIC by hydrodynamic calculations, assuming the existence of the quark gluon plasma 27.
} 


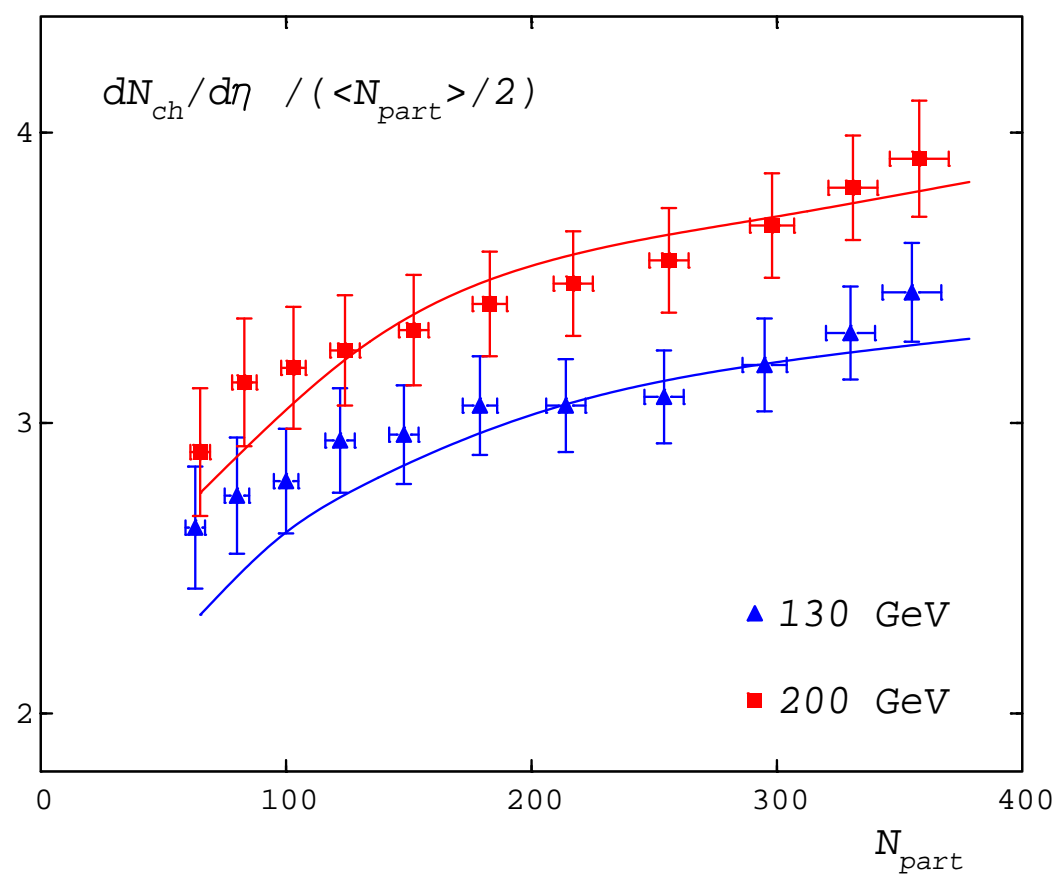

FIG. 2. The scaled pseudorapidity density for $|\eta|<1$ as a function of $N_{\text {part }}$ at $\sqrt{s}=130 \mathrm{GeV}$ and $200 \mathrm{GeV}$. Data are from the PHOBOS Collaboration, and curves from the "bottom-up" scenario, as described in the text.

In summary, the description provided by the "bottom-up" scenario is in agreement with RHIC data, provided the parameters $c$ and $c_{e q}$, which are not determined in the picture, lie in a given, limited range. In particular for $c \simeq 1$ and $c_{e q} \simeq 2$ the picture looks both reasonable and attractive. For these values the results (for the most central collisions) for $R, T_{e q}$ and $\tau_{e q}$ are 3, $230 \mathrm{MeV}$ and $3.6 \mathrm{fm}$, respectively, for $Q_{s}=1 \mathrm{GeV}$; for $Q_{s}=0.8 \mathrm{GeV}$, i.e. for $\mathrm{K}=1$, the values are $R=3, T_{e q}=210 \mathrm{MeV}$ and $\tau_{e q}=3.2 \mathrm{fm}$. However, the picture probably does not make much sense for a small value of $c \simeq 1 / 2$, which is currently favoured by numerical calculations of classical field equations, because the ratio $R$ turns out to become $R \simeq 6$, which is too much inelasticity when $Q_{s}=1 \mathrm{GeV}$. This discrepancy deserves further examination.

On the other hand, in order to accomodate the present RHIC data, the KLN description requires $c \simeq 3$. For the moment, due to a number of ambiguities, it is not yet clear whether this large value is compatible with the various constraints of the saturation picture.

The "bottom-up" scenario is likely to provide a more convincing agreement with data. However, this analysis will have to be supplemented by further ingredients, e.g. the calculation of $c_{e q}$, a more precise estimate of $c$, before one can finally claim a significant agreement with data.

Discussions with D. Kharzeev, M. Nardi, K. Redlich and R. Venugopalan are kindly acknowledged. RB acknowledges support, in part, by DFG, project FOR 339/2-1. The work of AHM is supported, in part, by a DOE Grant. The work of DTS is supported, in part, by a DOE Grant No. DOE-ER-41132 and by the Alfred P. Sloan Foundation. This research was supported in part by the National Science Foundation under Grant No. PHY99-07949; RB and DS thank ITP, UCSB, for kind hospitality, and for the facilities during the completion of this work.

[1] D. Kharzeev and M. Nardi, Phys. Lett. B 507 (2001) 121.

[2] D. Kharzeev and E. Levin, Phys. Lett. B 523 (2001) 79.

[3] D. Kharzeev, E. Levin and M. Nardi, hep-ph/0111315 (2001). 
[4] For a recent review, see D. Kharzeev, hep-ph/0204014 (2001).

[5] L. V. Gribov, E. M. Levin and M. G. Ryskin, Phys. Rep. 100 (1983) 1.

[6] A. H. Mueller and J. W. Qiu, Nucl. Phys. B 268 (1986) 427.

[7] J.-P. Blaizot and A. H. Mueller, Nucl. Phys. B 289 (1987) 847.

[8] L. McLerran and R. Venugopalan, Phys. Rev. D 49 (1994) 2233; Phys. Rev. D 49 (1994) 3352; Phys. Rev. D 50 (1994) 2225.

[9] A. Krasnitz and R. Venugopalan, Nucl. Phys. A 698 (2002) 209c, Plenary Talk by R. Venugopalan at QM2001, and references therein.

[10] For a recent review, e.g. L. McLerran, hep-ph/0202025 (2002).

[11] A. H. Mueller, Nucl. Phys. B 572 (2000) 227; Phys. Lett. B 475 (2000) 220.

[12] For a review on RHIC experiments, e.g. see J. L. Nagle, nucl-ex/0109016 (2001).

[13] B. B. Back et al. (PHOBOS Collaboration), Phys. Rev. Lett. 85 (2000) 3100; Phys. Rev. Lett. 88 (2002) 022302; nucl-ex/0201005 (2002).

[14] K. Adcox et al. (PHENIX Collaboration), Phys. Rev. Lett. 86 (2001) 3500; Phys. Rev. Lett. 87 (2001) 052301.

[15] I. G. Bearden et al. (BRAHMS Collaboration), Phys. Lett. B523 (2001) 227; nucl-ex/0112001 (2001).

[16] C. Adler et al. (STAR Collaboration), Phys. Rev. Lett. 87 (2001) 0112303.

[17] R. Baier, A.H. Mueller, D. Schiff and D. T. Son, Phys. Lett. B 502 (2001) 51.

[18] R. Baier, A.H. Mueller, D. Schiff and D. T. Son, Nucl. Phys. A 698 (2002) 217c, Talk given by D. T. Son at QM2001.

[19] A. H. Mueller, Talk given at "Statistical QCD", Bielefeld, August 2001, to appear in the Proceedings (Nucl. Phys. A).

[20] A. Krasnitz and R. Venugopalan, Phys. Rev. Lett. 86 (2001) 1717; A. Krasnitz, Y. Nara and R. Venugopalan, Phys. Rev. Lett. 87 (2001) 192302.

[21] Yu. V. Kovchegov, Nucl. Phys. A 692 (2001) 557.

[22] We acknowledge discussions with R. Venugopalan on the numerical evaluation of the "parton-liberation" coefficient $c$.

[23] Yu. L. Dokshitzer, V. A. Khoze, A. H. Mueller and S. I. Troyan, Basics of perturbative QCD, Editions Frontiérs, Gif-sur-Yvette, 1991, and references therein.

[24] J. Serreau and D. Schiff, JHEP 0111 (2001) 039.

[25] J. D. Bjorken, Phys. Rev. D 27 (1983) 140.

[26] F. Karsch, E. Laermann and A. Peikert, Nucl. Phys. B605 (2001) 579.

[27] D. Teaney, J. Lauret and E. V. Shuryak, Phys. Rev. Lett. 86 (2001) 4783; U. Heinz and P. F. Kolb, hep-ph/0204061, U. Heinz, Talk given at "Statistical QCD", Bielefeld, August 2001, to appear in the Proceedings (Nucl. Phys. A).

[28] K. Golec-Biernat and M. Wüsthof, Phys. Rev. D59 (1999) 014017; Phys. Rev. D60 (1999) 114023. 